\title{
La innovación educativa, subestimada: Análisis de la importancia que le otorgan los estudiantes de Periodismo
}

\author{
Sergio Roses \\ Universidad de Málaga \\ sergioroses@uma.es \\ María Luisa Humanes \\ Universidad Rey Juan Carlos \\ marialuisa.humanes@urjc.es
}

\begin{abstract}
Resumen:
Este artículo examina las actitudes de los estudiantes de Periodismo hacia la innovación educativa a partir de una encuesta transversal suministrada en siete países. El objetivo de la investigación es observar la importancia asignada por los estudiantes a la innovación educativa entre un conjunto de tareas académicas de sus profesores. Los estudiantes de periodismo españoles subestimaron la relevancia de la innovación educativa. Las mujeres otorgaron mayor importancia a la innovación educativa que los hombres. No se hallaron diferencias entre cinco universidades españolas.
\end{abstract}

Palabras clave: Innovación Educativa; Periodismo; Estudiantes universitarios; EEES; Actitudes; Innovación Docente

\section{Educational Innovation, Underestimated: Analysis of the Importance Given by Students of Journalism}

\begin{abstract}
:
This research examines the attitudes of Journalism students towards educational innovation with a cross-national survey in seven countries. The goal is to observe the importance given by students to the educational innovation among a set of tasks, which professors have to develop in their academic work. The Spanish Journalism students underestimated the relevance of educational innovation. Women gave more importance to innovation in education than men. No differences were found among five Spanish universities.
\end{abstract}

Key Words: Educational Innovation; Journalism; University Students, EHEA; Attitudes; Teaching innovation

Referencia normalizada:

Roses, S. y Humanes, M. L. (2014): La innovación educativa, subestimada: Análisis de la importancia que le otorgan los estudieantes de periodismo. Historia y Comunicación Social. Vol. 19. Núm. Especial Marzo. Págs. 479-490.

Sumario: 1. Introducción. 1.1. El punto de vista del profesorado. 1.2. El punto de vista de los estudiantes. 2. Metodología. 3. Resultados. 3.1. Importancia otorgada a la innovación educativa. 3.2. Diferencias entre estudiantes españoles. 3.3. Diferencias con otros países. 4. Conclusiones. 5. Referencias bibliográficas. 


\section{Introducción}

Estudios recientes han demostrado que la valoración de los estudiantes sobre la formación recibida en las facultades de Periodismo no supera el aprobado raspado (Humanes y Roses, 2014), de manera que la calidad de la docencia impartida sigue considerándose "regular", una estima que se ha mantenido constante desde los 70 hasta la actualidad entre los egresados de las facultades (Gómez y Roses, 2013). A pesar de las políticas de innovación educativa implementadas en las facultades de Periodismo, la valoración de los estudiantes sobre el trabajo de sus docentes pone en entredicho los posibles efectos benévolos de la innovación realizada hasta el momento.

Desde el punto de vista de los alumnos, los docentes de periodismo desarrollan una amplia variedad de actividades en las facultades: estimulan el pensamiento crítico de los estudiantes, realizan investigaciones científicas, publican, promueven las prácticas en empresas, comparten su conocimiento...Tres de esas actividades componen lo que Mellado y Subervi (2012) denominaron el didactic-oriented role, actitud profesional compartida por aquellos profesores que dan mucha importancia a desarrollar habilidades didácticas, a innovar en las prácticas docentes y a evaluar constantemente los métodos de enseñanza utilizados. Habida cuenta de que la innovación educativa es importante para un amplio sector del profesorado, esta investigación aborda de manera exploratoria una cuestión habitualmente olvidada: las actitudes de los estudiantes de Periodismo hacia la innovación educativa en sí misma.

\subsection{El punto de vista del profesorado}

La innovación o la renovación de las técnicas docentes es una actividad tan vieja como la propia enseñanza. Allá donde hay un docente responsable y comprometido con el aprendizaje de sus estudiantes, hay simiente para la investigación-más o menos sofisticada - y la averiguación de métodos o dinámicas más efectivas o convenientes. Por tanto, la innovación ha sido una cuestión relevante para el profesorado más motivado desde la génesis de la educación superior. No obstante, además del prurito profesional, el profesorado ha contado con otras fuentes de motivación. Al igual que otros académicos (e.g. Ortiz Sobrino, 2009; Farias et al., 2010), no negaremos que, para el profesorado universitario de la titulación de Periodismo, el Espacio Europeo de Educación Superior (EEES) supone el empuje global e institucional hacia la consciencia de la importancia que ocupa la innovación educativa en su labor docente.

El EEES ha dado lugar, sobre todo, a la sistematización de programas y proyectos de innovación que habrían de transformar tanto la función del profesorado como el proceder y objetivos de los estudiantes. Las experiencias han proliferado en la docencia del Periodismo, tal y como puede observarse en la literatura (e.g. Peinado et al., 2011; Vicente y Clua, 2009; Ruiz y Guerrero, 2012; entre muchos otros), de manera que algunos docentes llevan casi una década aplicando de forma continuada metodologías innovadoras (e.g. Bueno y Hanninen, 2012). 
Las políticas institucionales han ayudado a que gran parte del profesorado universitario se haya volcado con la innovación educativa: el Ministerio de Educación, las Comunidades Autónomas y las propias universidades han desarrollado programas de incentivos para la realización de proyectos de innovación. Asimismo, las agencias evaluadoras de la calidad del profesorado (e.g. ANECA) y las universidades han incluido el requisito de haber participado en proyectos de innovación educativa en los pliegos que han de cumplir los candidatos para promocionar o ser contratados. En consecuencia, el profesorado de la titulación de Periodismo, al igual que el de otras disciplinas, ha movilizado recursos para alcanzar los méritos necesarios: proyectos financiados por terceros o autofinanciados, publicaciones, congresos, revistas... Dicho esto, se puede colegir la importancia que tiene hoy para el profesorado su actividad relacionada con la innovación educativa.

\subsection{El punto de vista de los estudiantes}

Hasta el momento, los estudios empíricos sobre innovación docente que tienen a los estudiantes como protagonistas se han centrado en la "satisfacción" o en la "percepción" de los alumnos sobre determinadas técnicas o experiencias innovadoras concretas. Es decir, habitualmente, como parte de los proyectos de innovación, se solicitan informes, o se entrevista o encuesta a los alumnos sobre sus preferencias o actitudes acerca de las innovaciones a las que han sido expuestos o pueden ser expuestos en el futuro. Por ejemplo, Cabero et al. (2010) e Hinojo et al. (2009) examinaron la satisfacción y percepción de estudiantes sobre el blended learning; Gómez, Roses y Farias (2012) exploraron las actitudes hacia el uso académico de las redes sociales; Alcolea y Pérez (2012) observaron la valoración de los estudiantes sobre la acción tutorial (2012); Sabés y Carniel (2012) pidieron a los estudiantes que opinaran sobre un método innovador de enseñanza de redacción periodística; Roca-Cuberas et al. (2010) o García Ureta et al. (2012) han evaluado la efectividad de la enseñanza por competencias; otros se han preocupado por evaluar la calidad en sí de la innovación, como Mauri et al. (2007) que plantearon un método de evaluación constructivista; otros han investigado las percepciones y preferencias de los alumnos en relación a la utilización de distintas metodologías (e.g., Pastor y Vargas, 2009; Martín Peña et al., 2011; etcétera). Sin embargo, en nuestra revisión de la literatura, no hallamos estudios que ahonden en qué piensan los estudiantes de periodismo españoles sobre la importancia que tiene la innovación educativa en sí en el marco de las funciones de sus profesores.

En esta investigación, nos planteamos cuál es la importancia que dan los estudiantes de periodismo a dichas atribuciones de sus docentes. Se trata, por tanto, de dar respuesta a las siguientes preguntas de investigación:

Primera (RQ1), ¿qué importancia asignan los estudiantes a la innovación educativa entre un conjunto de tareas académicas que habitualmente realizan sus profesores? 
Segunda (RQ2), ¿existen diferencias en la importancia otorgada a la innovación educativa según "género", "curso académico" y "Universidad", entre los estudiantes españoles?

Tercera (RQ3), ¿existen diferencias entre los estudiantes españoles y de otros países en la importancia otorgada a la innovación educativa? Para ello se comparan dichas actitudes con las de estudiantes de Periodismo de otros seis países.

\section{Metodología}

Se empleó una encuesta transnacional suministrada en siete países (i.e. Australia, Brasil, Chile, España, México, Suiza y Estados Unidos), puesto que este estudio forma parte de una investigación comparada titulada Journalism Sudents Proyect (Mellado et al., 2012). En el caso español, la población la constituyeron los estudiantes de la titulación de Periodismo en el curso 2011-12. Usamos un criterio de conveniencia para la selección de las universidades basado en nuestra red académica: U. Complutense de Madrid, U. Rey Juan Carlos, U. de Sevilla, U. de Málaga y U. Jaume I de Castellón. La encuesta se realizó a todos los cursos. Solo no la cumplimentaron quienes estaban desinteresados en participar o quienes no se encontraban en la clase cuando se suministró. Se recabaron 1,552 cuestionario completos. El cuestionario incluía un set de 16 ítems, basado en un estudio previo de Mellado y Subervi (2012), que mide la importancia (Nada importante=1, Poco importante=2, Algo importante $=3$, Muy importante $=4$, Extremadamente importante $=5$ ) concedida a una serie de tareas académicas que habitualmente realizan los profesores de Periodismo. Entre dichas labores, se encuentran tres cuestiones que están relacionadas con la práctica de la innovación educativa. A saber, "Innovar en las prácticas docentes", ítem que se refiere explícitamente a la renovación de las técnicas pedagógicas; "Evaluar constantemente los métodos docentes empleados", ítem que hace hincapié en el obligado diagnóstico de la actividad y experiencias docentes que conlleva la innovación, y "Manejar habilidades y técnicas docentes", cuestión que puede entenderse como la consecuencia o resultado de incorporar la innovación docente en el día a día del profesor universitario.

Para resolver RQ1, se usaron técnicas de estadística descriptiva (medidas de tendencia central y dispersión). En el caso de RQ2 y RQ3, empleamos la prueba T de Student y ANOVA de un factor, según la naturaleza de la variable independiente. Asimismo, nos servimos de regresiones lineales múltiples para confirmar los resultados de RQ2. El análisis de datos se realizó con SPSS. 


\section{Resultados}

\subsection{Importancia otorgada a la innovación educativa}

¿Qué importancia asignan los estudiantes españoles a la innovación educativa entre un conjunto de tareas académicas que habitualmente realizan sus profesores? (RQ1). Los resultados indicaron que, aunque los estudiantes consideran bastante importantes las actividades de sus docentes relacionadas con la innovación educativa, no las incluyen entre las tareas más relevantes. Los estudiantes atribuyeron mucha importancia a cuestiones como "Desarrollar un proceso de pensamiento crítico y reflexivo" $(\mathrm{M}=4,43$. DT= 0,850$)$; "Priorizar la práctica como lo fundamental dentro de la formación del periodismo" $(\mathrm{M}=4,33$. $\mathrm{DT}=0,892)$; "Promover las prácticas periodísticas bajo condiciones del mundo real" $(\mathrm{M}=4,29$. DT=0,861); "Compartir conocimiento" $(\mathrm{M}=4,23$. DT= 0,807); "Construir nuevo conocimiento" $(\mathrm{M}=$ 4,21 . DT= 0,840); "Acentuar el dominio de técnicas profesionales en el currículum" $(\mathrm{M}=4,03$. $\mathrm{DT}=0,918)$; "Manejar habilidades y técnicas docentes" $(\mathrm{M}=4,01$. DT= 0,856 - - he aquí el primero de los ítems relacionados con la innovación docente-, $\mathrm{y}$ "Explicar fenómenos sociales a través del análisis" $(M=3,92$. DT=0,913).

Los alumnos emplazaron en un segundo nivel de importancia otras tareas como "Desarrollar investigación académica" $(\mathrm{M}=3,66$. DT= 1,005); "Presentar y promover el trabajo científico entre la academia" $(\mathrm{M}=3,44$. DT=0,967); "Publicar material científico" $(\mathrm{M}=3,32$. DT= 0,999) y "Enfatizar la teoría en la formación" $(\mathrm{M}=3,16$. $\mathrm{DT}=1,072)$. Precisamente, dos de las tareas de los profesores relacionadas con la innovación educativa pertenecen a este segundo nivel de relevancia. De entre las 14 actividades planteadas, "Innovar en las prácticas docentes" resultó solamente la novena en importancia $(\mathrm{M}=3,79$. $\mathrm{DT}=1,002)$, mientras que "Evaluar constantemente los métodos docentes empleados" $(\mathrm{M}=3,51 . \mathrm{DT}=1,042)$ fue la undécima.

\subsection{Diferencias entre los estudiantes españoles}

¿Existen diferencias en la importancia otorgada a la innovación educativa según "género", "curso académico" y "Universidad", entre los estudiantes españoles? (RQ2). Los resultados arrojaron diferencias significativas entre géneros. Las mujeres consideraban más importante $(\mathrm{M}=3,89$. $\mathrm{DT}=0,971)$ "Innovar en las prácticas docentes" que sus compañeros hombres $(\mathrm{M}=3,61$. DT=1,032) $[\mathrm{t}(1065,298)=-5,156 . \mathrm{p}<$ $0,05]$. Asimismo, las féminas otorgaban significativamente más importancia $(\mathrm{M}=$ 3,55. DT = 1,056) a "Evaluar constantemente los métodos docentes empleados" que los varones $(\mathrm{M}=3,44$. $\mathrm{DT}=1,011)[\mathrm{t}(1532)=-2,027 . \mathrm{p}<0,05]$. La tendencia se repitió también en las actitudes referidas a "Manejar habilidades y técnicas docentes". Para las alumnas, esta cuestión era más importante $(\mathrm{M}=4,09$. $\mathrm{DT}=0,823)$ que para los hombres $(\mathrm{M}=3,85$. DT $=0,893)[\mathrm{t}(1041,693)=-5,280 . \mathrm{p}<0,05]$.

Por otra parte, hallamos diferencias significativas en la importancia atribuida a "Innovar en las prácticas docentes" según el curso del estudiante $[\mathrm{F}(2,623,055)=$ 
4,965. $p<0,05]$, habiendo varianzas desiguales entre los grupos. Las pruebas post hoc T3 de Dunnett $(p<0,05)$ confirmaron que los alumnos de los cursos intermedios le otorgaban significativamente menos importancia $(\mathrm{M}=3,73)$ que sus compañeros del primer $(\mathrm{M}=3,90)$ y del último curso $(\mathrm{M}=3,85)$.

También encontramos diferencias significativas en la relevancia concedida a "Manejar habilidades y técnicas docentes" según el curso del estudiante $[F(2,9,044)=$ 6,223. $\mathrm{p}<0,05]$, habiendo varianzas iguales entre los grupos. Las pruebas post hoc HSD de Tukey $(\mathrm{p}<0,05)$ confirmaron que los alumnos del primer curso de la carrera daban significativamente mayor importancia $(M=4,13)$ a esta cuestión que los estudiantes de cursos intermedios $(\mathrm{M}=3,96)$ y del último curso $(\mathrm{M}=3,96)$.

No se hallaron diferencias significativas en la importancia atribuida a "Evaluar constantemente los métodos docentes empleados" según el curso del estudiante. Asimismo, la prueba ANOVA de un factor resultó no significativa en los tres casos para la variable "Universidad", por consiguiente, independientemente de la universidad española en la que estuvieran estudiando, los alumnos encuestados otorgaron en promedio la misma importancia a las tres cuestiones analizadas.

Realizamos, por último, un análisis de regresión lineal múltiple para examinar simultáneamente el efecto del género y del curso del estudiante en la importancia que le otorgaban a "Innovar en las prácticas docentes". El modelo resultó significativo $[\mathrm{F}(2,1518)=15,259 . \mathrm{p}<0,001]$, si bien la explicación de la varianza fue extremadamente baja $\left(\mathrm{R}^{2}=0,020\right)$. Observados los coeficientes de regresión estandarizados, hallamos que solo "género" $[\beta=-0,137$. $\mathrm{t}(1518)=5,396 . \mathrm{p}<0,001]$ resultó un predictor estadísticamente significativo, lo que indica que las mujeres tendieron a otorgar mayor importancia que los hombres a "Innovar en las prácticas docentes", independientemente del curso académico y de la universidad en la que estudiaran.

Utilizamos el mismo procedimiento para las otras dos variables dependientes. No obtuvimos resultados significativos en el caso de la importancia concedida a "Evaluar constantemente los métodos docentes empleados". Por tanto, los estudiantes españoles otorgaron en promedio la misma relevancia a esta cuestión, independientemente de su género, curso académico y universidad.

El modelo resultó estadísticamente significativo para la importancia atribuida a "Manejar habilidades y técnicas docentes" $[\mathrm{F}(2,1515)=20,393 . \mathrm{p}<0,001]$. De nuevo la explicación de la varianza fue extremadamente baja $\left(\mathrm{R}^{2}=0,026\right)$. El análisis de los coeficientes beta tipificados indicó que los dos predictores propuestos eran estadísticamente significativos, siendo el género $[\beta=-0,143$. $t(1515)=-5,621 . p<0,001]$ más importante que el curso académico $[\beta=-0,081 . t(1515)=-3,187 . p<0,001]$. Esto quiere decir que las mujeres y quienes estudian en los primeros cursos académicos consideraron más importante "Manejar habilidades y técnicas docentes" que el resto, independientemente de la universidad donde estuvieran matriculados. 


\subsection{Diferencias con otros países}

Por último, ¿existen diferencias entre los estudiantes españoles y de otros países en la importancia otorgada a la innovación educativa? (RQ3). Los resultados indicaron la existencia de diferencias significativas en la importancia asignada a "Innovar en las prácticas docentes" según el país donde estudia el alumno $[\mathrm{F}(6,1048,829)=35,839$. $\mathrm{p}<0,05]$, habiendo varianzas desiguales entre los grupos. Las pruebas post hoc T3 de Dunnett $(p<0,05)$ confirmaron que existían diferencias significativas entre el parecer de los estudiantes españoles $(M=3,79)$ y otros cuatro países. En este caso, los estudiantes de Chile $(M=4,23)$, México $(M=4,07)$ y Brasil $(M=3,96)$ consideraban esa tarea significativamente más importante que los españoles. Al otro extremo, se ubicaban los estudiantes suizos $(M=3,35)$, a quienes les importaba significativamente menos que a los españoles la innovación de las prácticas docentes. El análisis indicó también que los estudiantes españoles tenían una actitud semejante en términos estadísticos a la de sus compañeros de Australia $(\mathrm{M}=3,77)$ y Estados Unidos $(\mathrm{M}=3,61)$.

Hallamos también diferencias significativas entre países en la importancia atribuida a "Evaluar constantemente los métodos docentes empleados" $[\mathrm{F}(6,1043,458)=$ $35,839$. $p<0,05]$, habiendo varianzas desiguales entre los grupos. Las pruebas post hoc T3 de Dunnett $(p<0,05)$ confirmaron que existían diferencias significativas entre la opinión de los españoles $(M=3,51)$ y la de otros cuatro países. Los alumnos de Australia $(M=3,76)$, Brasil $(M=3,95)$, México $(M=4,01)$ y Chile $(M=4,20)$ otorgaban significativamente más importancia a esta actividad que los estudiantes españoles que, en este caso, mantenían un parecer similar en términos estadísticos al de los alumnos de Suiza y Estados Unidos.

Por último, encontramos diferencias significativas entre países en la importancia dada a "Manejar habilidades y técnicas docentes" $[\mathrm{F}(6,1038,913)=40,871 . \mathrm{p}<0,05]$, habiendo varianzas desiguales entre los grupos. Las pruebas post hoc T3 de Dunnett $(\mathrm{p}<0,05)$ confirmaron que existían diferencias significativas entre la opinión de los españoles $(M=4,01)$ y el resto de países estudiados. Los estudiantes de México ( $M=$ $4,16)$, Chile $(M=4,24)$, Australia $(M=4,40)$ y Suiza $(M=4,44)$ daban significativamente más importancia a este aspecto que los españoles. Y, al contrario, los alumnos de Brasil $(M=3,85)$ y Estados Unidos $(M=3,47)$ otorgaban significativamente menos importancia que los españoles al ítem.

\section{Conclusiones}

Antes de enunciar las conclusiones más significativas, es preciso referirse al alcance de la investigación aquí relatada. Cabe destacar que se ha contado con la mayor muestra de estudiantes utilizada hasta la fecha en estudios sobre Periodismo en España, incluyendo todos los cursos de la titulación tanto de licenciatura como de grado. Igualmente, se trata de una investigación pionera en el estudio de las percep- 
ciones de los alumnos de Periodismo sobre la innovación docente. Por consiguiente, los datos previamente descritos y las conclusiones reseñadas a continuación constituyen un corpus valioso para estudios futuros. Los resultados nos sugieren recomendar a los responsables académicos de las facultades y departamentos que valoren la necesidad de organizar, coordinar y regular el tipo y la cantidad de experiencias de innovación que se desarrollan en las aulas. Asimismo, recomendamos que se incida en acciones de comunicación sobre los estudiantes en las que se divulgue la utilidad e importancia de la innovación educativa como parte sustancial de la actividad docente. Las conclusiones que dan lugar a los razonamientos que se han expuesto hasta el momento se enuncian y discuten en las líneas siguientes:

Los estudiantes de Periodismo españoles subestiman la importancia de la innovación educativa. La "innovación" de las prácticas docentes o la "evaluación" de los métodos empleados son ubicadas en el furgón de cola de las actividades de los profesores, de acuerdo con la importancia atribuida por los estudiantes. Consideran "algo importantes" las actividades vinculadas a la innovación, pero no las incluyen entre las tareas más relevantes que deben atender sus profesores. Sin embargo, llama la atención el hecho de que "Manejar habilidades y técnicas docentes" sí se considere una tarea "muy importante" del profesorado. Precisamente, el objeto de este ítem, el dominio de la pedagogía y la didáctica, entraña de alguna manera "innovar" y "evaluar". Podemos ofrecer dos explicaciones alternativas para esta disparidad en la relevancia atribuida las variables del estudio:

a) Debido a la exigencia de experiencia en innovación educativa entre los requisitos de las acreditaciones y de los baremos de contratación del profesorado, se han multiplicado los pequeños estudios y aplicaciones experimentales, muchos de ellos realizados de manera descoordinada con otros estudios o con el desconocimiento de los centros. De esta manera, sin que medie control ni regulación alguna sobre cuántas y qué experiencias pueden realizarse sobre un grupo determinado de estudiantes, queda a discreción del profesorado-ávido de méritos y resuelto a poner en práctica sus propias tesis. Quizás las actitudes de los estudiantes de nuestro estudio están reflejando hastío hacia la proliferación de experiencias de innovación a las que son expuestos a lo largo de su paso por las aulas, sin que eso menoscabe el reconocimiento y aprecio de la pericia y formación docente de sus profesores.

b) Habitualmente, se informa a los alumnos de que son sujetos participantes en un proyecto de innovación educativa, pero es muy poco frecuente informar a los estudiantes ordinarios del siguiente curso de que la actividad docente o las técnicas que se están empleando surgieron de un proceso de innovación y evaluación en el marco de un proyecto. Existe la posibilidad de que los estudiantes consideren las experiencias innovadoras y sus evaluaciones como actividades que reportan rédito exclusivamente a sus profesores. En tal caso, los estudiantes estarían percibiendo los proyectos de innovación como algo ajeno a ellos, que no tiene efecto real sobre su formación.

Independientemente de cuál sea la interpretación más acertada de los resultados, sería conveniente dar cuenta al alumnado de que las buenas prácticas docentes son 
fruto, en muchas ocasiones, de la innovación educativa. Asimismo, sería recomendable que las actividades de innovación docente fueran planificadas en el ámbito de los centros o de los departamentos en aras de no saturar a los sujetos experimentales, al tiempo de plantear estudios más amplios con objetivos más exigentes.

A la luz de los datos del estudio, las actitudes hacia la innovación educativa varían poco entre los estudiantes españoles; por tanto, podemos pensar que el fenómeno se da de manera similar en todo el territorio español. Si bien la capacidad predictiva de los modelos testeados fue exigua, puede afirmarse que la importancia otorgada a los ítems referidos a la innovación no varía según universidades. Apenas hay diferencias entre cursos aunque los estudiantes de los primeros niveles atribuyeron mayor importancia a que los profesores manejaran habilidades y técnicas docentes. Tales estudiantes se enfrentan por vez primera a la Universidad, donde han de profundizar en materias de mayor calado que en Bachillerato. Quizás por esa intranquilidad que se atempera a medida que avanzan en la carrera otorgan más valor a la capacidad de enseñar de sus profesores. Las diferencias individuales parecen explicarse sobre todo a partir del género del estudiante. Las mujeres valoran más que los hombres la "innovación" en las prácticas y el dominio de habilidades docentes. Resulta complicado presentar una explicación racional a las disimilitudes entre hombres y mujeres en esta cuestión. Quizás pueda atribuirse a las diferencias, sugeridas por los neurocientíficos, que existen en el cerebro de ambos sexos (Rubia, 2007), tópico que, sin embargo, excede al conocimiento de los autores de este artículo.

Por último, los estudiantes españoles destacan por otorgar menos importancia a la innovación educativa que la mayoría de los países estudiados. De hecho, en los tres ítems analizados, los españoles se encuentran entre los alumnos de alguno de los tres países que menos relevancia atribuyen al aspecto específico de la innovación educativa que plantea el ítem. Las mayores diferencias se hallaron con los estudiantes de Chile y México que, en conjunto, dan mayor trascendencia a la innovación que el resto de países. Las actitudes de los españoles fueron más similares a las de los estudiantes de EEUU, y en menor medida también a la de australianos y suizos, aunque con algunas diferencias en ítems concretos. Explicar las valoraciones de los estudiantes de cada uno de los países encuestados excede a este trabajo, sin embargo, podemos apuntar una aparente diferencia entre países occidentales y latinoamericanos, acaso con Brasil a medio camino. Quizás la semejanza con EEUU apoye la hipótesis de que los estudiantes que son expuestos a una sobreabundancia de experiencias innovadoras, desarrollan hastío por estas actividades, considerándolas poco relevantes entre las tareas de sus profesores.

En definitiva, volviendo al caso español, los datos del estudio permiten esclarecer que verdaderamente los estudiantes españoles conceden a la innovación educativa una trascendencia inferior a la que dispensa el sistema educativo universitario español en su conjunto; esto es, las instituciones y el profesorado. Obviamente, algo falla si los principales beneficiarios de la innovación educativa subestiman su importancia. En los próximos años, será preciso continuar tomando el pulso a las actitudes de los alumnos sobre este objeto. Los futuros desarrollos de esta investigación deberán 
construir instrumentos de medición más complejos, así como indagar en la identificación de predictores que permitan desarrollar modelos predictivos más precisos.

\section{Bibliografía}

ALCOLEA DÍAZ, G. y PÉREZ SERRANO, M.-J. (2012). “Un paso más allá en la planificación docente universitaria: el plan de acción tutorial”. En: Estudios sobre el Mensaje Periodístico, $\mathrm{n}^{\circ} 18$. p. 45-55. [23-08-2013] [Disponible en: http:// revistas.ucm.es/index.php/ESMP/article/view/40889/39147].

BUENO, T. y HANNINEN, L. (2012). "Innovación docente con jóvenes interactivos: proyectos sociales reales y estrategias digitales para enseñar comunicación”. En: Estudios sobre el Mensaje Periodístico, n¹8. p. 161-170. [22-08-2013] [Disponible en: http://revistas.ucm.es/index.php/ESMP/article/view/40892/39174].

CABERO, J.; LLORENTE, M.-C. y PUENTES, A. (2010). "La satisfacción de los estudiantes en red en la formación semipresencial". En: Comunicar, n ${ }^{\circ} 35$. p. 149-157. [06-05-2012] [Disponible en: http://www.revistacomunicar.com/ index.php? contenido $=$ detalles \&numero $=35 \&$ articulo $=35-2010-18 \&$ mostrar $=$ comocitar\#comocitar].

FARIAS, P.; GÓMEZ AGUILAR, M. y PANIAGUA, F.-J. (2010). "Panorama de los estudios de periodismo en España ante el Espacio Europeo de Educación Superior". En SIERRA SÁNCHEZ, J. (2010). Los estudios de Ciencias de la Comunicación en el EEES. Madrid: Fragua.

GARCÍA URETA, I.; TORAL, G. y MURELAGA, J. (2012). "Propuesta docente para la formación de comunicadores: desarrollo de competencias psicológicas y conexión con los retos profesionales". En: Estudios sobre el Mensaje periodístico, no 18. p. 413-423. [22-08-2013] [Disponible en: http://revistas.ucm.es/index.php/ ESMP/article/view/40996/39246].

GÓMEZ AGUILAR, M.; ROSES, S. y FARIAS, P. (2012). "El uso académico de las redes sociales en universitarios". En Comunicar, no 38. P. 131-138. [04-09-2013] [Disponible en: http://goo.gl/z60ESg].

GÓMEZ CALDERÓN, B. y ROSES, S. (2013). "Valoración de los profesionales sobre la enseñanza del periodismo en España. Un análisis intergeneracional". En: Estudios sobre el Mensaje Periodístico, vol. 19, no 1. [04-09-2013] [Disponible en: http://revistas.ucm.es/index.php/ESMP/article/view/42529].

HINOJO, F.-J.; AZNAR, I. y CÁCERES, M.-P. (2009). "Percepciones del alumnado sobre el blended learning en la universidad". En: Comunicar, no 33. p. 165-174. [06-06-2013] [Disponible en: http:/www.revistacomunicar.com/index.php?contenido $=$ detalles $\&$ numero $=33 \&$ articulo $=33-2009-20 \&$ mostrar $=$ comocitar $\#$ comocitar].

HUMANES, M. L. y ROSES, S. (2014-en prensa). "Valoración de los estudiantes sobre la enseñanza del periodismo en España". En: Comunicar, no 42. [Disponible a partir de 2014 en: www.revistacomunicar.es]. 
MARTÍN PEÑA, M.-L.; DÍAZ, E.; CASTILLO, B. y DEL BARRIO, L. (2011). "Estudio comparativo de cambios metodológicos y percepción del alumno en la materia de Dirección de producción y operaciones para la adquisición de competencias en el proceso de adaptación al EEES”. En: Revista de Formación e Innovación Educativa Universitaria, Vol. 4, nº 2. p. 126-144. [23-08-2013] [Disponible en: http://webs.uvigo.es/refiedu/Refiedu/Vol4_2/REFIEDU_4_2_5.pdf].

MAURI, T.; COLL, C. y ONRUBIA, J. (2007). "La evaluación de la calidad de los procesos de innovación docente universitaria. Una perspectiva constructivista". En: Red U. Revista de Docencia Universitaria, $\mathrm{n}^{\circ}$ 1, 3. [22-10-2011] [Disponible en: http://www.redu.um.es/Red_U/1/].

MELLADO, C. y SUVERBI, F. (2012). "Mapping educational role dimensions among chilean Journalism and Mass Communication educators". En: Journalism Practice, $\mathrm{n}^{\mathrm{o}}$ 105. [01-08-2013] [Disponible en: DOI:10.1080/17512786.2012.71 8584].

ORTIZ SOBRINO, M.-A. (2009). "La reconversión de la formación en comunicación desde el Espacio Europeo de Educación Superior". En: Revista Icono14, n¹4. p. 35-49. [03-09-2013] [Disponible en: http://www.icono14.net].

PASTOR, G. y VARGAS, M. (2009). "Los métodos de innovación docente. Perspectiva de los estudiantes". En: e-pública, Revista electrónica sobre la enseñanza de la Economía Pública, n5. p. 58-90. [23-08-2013] [Disponible en: http://xurl. es/0ovug].

PEINADO, M.; FERNÁNDEZ-SANDE, M.; ORTIZ SOBRINO, M.A., y RODRÍGUEZ BARGO, M.-D. (2011). "Hacia un aprendizaje activo de la empresa informativa en el EEES. aplicación del podcasting y otras herramientas de comunicación". En: Razón y Palabra, nº75. [03-09-2013] [Disponible en: http://goo. $\mathrm{g} 1 / 6 \mathrm{zbVlm}]$.

ROCA-CUBERES, C.; GUERRERO-SOLE, F.; RODRIGO-ALSINA, M.; ALMIRON, N. y GÓMEZ, L. (2010). "El aprendizaje por competencias en el EEES: el caso de la asignatura de Teorías y Métodos de Investigación de la Comunicación en la UPF”. En SIERRA SÁNCHEZ, J. y CABEZUELO, F. (coord.), (2010). Competencias y perfiles profesionales en los estudios de ciencias de la comunicación. Madrid: Fragua.

RUBIO, F.-J. (2007). El sexo del cerebro: La diferencia fundamental entre hombres y mujeres. Madrid: Temas de Hoy.

RUIZ, M.-J. y GUERRERO, D. (2012). "El desarrollo de competencias profesionales dentro del marco del EEES. El caso de los grados en Comunicación". En Estudios sobre el Mensaje Periodístico, $\mathrm{n}^{\mathrm{o}}$ 18. [03-09-2013] [Disponible en: http://revistas. ucm.es/index.php/ESMP/article/view/40962].

SABÉS, F. y CARNIEL, R. (2012). "Metodología para la enseñanza de la Escritura en Prensa en el Grado de Periodismo de la UAB". En: @tic, revista d'innovació educativa, $\mathrm{n}^{\circ}$ 9. [03-09-2013] [Disponible en: http://goo.gl/hUXEWF].

VICENTE, M. y CLUA, A. (2009). "La docencia de Teoría de la Comunicación y la Información en entornos virtuales: la UOC como experiencia pionera". En: 
Diálogos de la Comunicación, n78. [03-09-2013] [Disponible en: http://goo.gl/ ngxqoH].

\section{Los autores}

Sergio Roses es profesor en el Departamento de Periodismo de la Universidad de Málaga y consultor en Comunicación. Autor de una docena de artículos sobre la profesión periodística, la credibilidad de los medios y la docencia universitaria en Periodismo en revistas como Journalism Studies, Comunicación y Sociedad, Estudios sobre el Mensaje Periodístico, Revista Comunicar, Revista Latina de Comunicación Social o Revista Observatorio OBS, entre otras. Coautor de un capítulo en "The Global Journalist in the 21st Century", editado por Routledge y dirigido por David H. Weaver y Lars Wilnat, ha sido colaborador del Informe Anual de la Profesión Periodística entre 2006 y 2011.

$\mathrm{M}^{\mathrm{a}}$ Luisa Humanes es profesora Titular de Universidad en el área de conocimiento de Comunicación Audiovisual y Publicidad. Tiene reconocidos dos sexenios de investigación por la CNEAI (1997-2009). Ha formado parte de la Comisión Delegada de la Facultad de CC. de la Comunicación de la Universidad Rey Juan Carlos para la reforma de las titulaciones de Comunicación en el marco del EEES. Miembro vocal de la Comisión de Política Científica de AE-IC, sus líneas de investigación son: Teorías y métodos de investigación aplicados a la comunicación, profesionalización y culturas profesionales en el periodismo y análisis del discurso periodístico. 